\title{
Multi-Product Duopoly With Cross-Product Cost Interdependencies
}

\section{Citation}

Biglaiser, Gary, and Andrei Hagiu. "Multi-Product Duopoly With Cross-Product Cost

Interdependencies." Harvard Business School Working Paper, No. 16-010, July 2015.

\section{Permanent link}

http://nrs.harvard.edu/urn-3:HUL.InstRepos:17541633

\section{Terms of Use}

This article was downloaded from Harvard University's DASH repository, and is made available under the terms and conditions applicable to Open Access Policy Articles, as set forth at http:// nrs.harvard.edu/urn-3:HUL.InstRepos:dash.current.terms-of-use\#OAP

\section{Share Your Story}

The Harvard community has made this article openly available.

Please share how this access benefits you. Submit a story.

\section{Accessibility}


H A R VAR D

\title{
Multi-Product Duopoly With Cross-Product Cost Interdependencies
}

\author{
Gary Biglaiser \\ Andrei Hagiu
}

\section{Working Paper}

16-010

July 21, 2015 


\title{
Multi-Product Duopoly With Cross-Product Cost Interdependencies
}

\author{
Gary Biglaiser*and Andrei Hagiu ${ }^{\dagger}$
}

July 21, 2015

\begin{abstract}
Many multi-product firms incur a complexity fixed cost when offering different product lines in different quality tiers relative to the case when offering all products lines in the same quality tier (high or low). Such fixed costs create an interdependency between firms' choices of quality tiers across different product lines, even when demands are independent. We investigate the effects of this interdependency on equilibrium profits in a Stackelberg duopoly game. Both firms' profits are (weakly) higher when the complexity cost is infinite than when it is 0. The Stackelberg leader's profits are always (weakly) higher with a positive complexity fixed cost, but its profits can be nonmonotonic in the magnitude of this cost. The Stackelberg follower's profits can be lower when the complexity fixed cost is positive than when it is equal to 0 .
\end{abstract}

JEL Classifications: L1, L2, L8

Keywords: multi-product duopoly, vertical differentiation, fixed costs.

\section{Introduction}

In many industries, multi-product firms do not have full flexibility to choose different quality tiers for different product categories (lines). Once committed to a certain quality tier (high or low) in one product line, it is usually more costly to offer another product line in a different quality tier instead of offering it in the same tier. This is one of the main reasons for which many multi-product firms in various industries typically offer a consistent product and service quality for most of their product categories. Examples include retailers (e.g. Neiman Marcus, Saks Fifth Avenue at the highend vs. T.J. Maxx, Wal-Mart at the low-end), e-commerce (e.g. Eastbay and Zappos selling shoes and clothing with a high level of customer service vs. eBay relying on third-party sellers with typically lower-quality service), furniture stores (e.g. Crate \& Barrel at the mid- to high-end vs. Ikea at

\footnotetext{
${ }^{*}$ University of North Carolina, gbiglais@email.unc.edu

${ }^{\dagger}$ Harvard Business School, Boston, MA 02163, E-mail: ahagiu@hbs.edu
} 
the low-end), airlines (full-price carriers such as American Airlines, Emirates, United vs. low-cost carriers such as EasyJet, Ryan Air, Southwest), etc. Some companies have managed to maintain different quality tiers for different product lines, e.g. Sears (low-end in clothing, but lower-upper end in tools), Amazon (retailer with high level of service on some products, but eBay-like marketplace on other products), Singapore Airlines (high-end airline, but also operates Scoot, a low-cost carrier, on some, typically shorter, routes). ${ }^{1}$ However, other companies' attempts to do so have failed, e.g. J.C. Penney's unsuccessful attempt to become an upscale department store between $2011-2013,{ }^{2}$ the failure of Delta's low-cost carrier Song, ${ }^{3}$ etc. These examples illustrate that in many circumstances there are diseconomies of scope when offering multiple quality tiers on different product lines or categories. Such diseconomies can stem from a combination of brand stickiness - it is difficult to cover multiple quality tiers with the same brand or operate multiple brands in the same organization - and operational complexity - different quality tiers have different internal processes (see Rivkin, 2006).

We analyze a Stackelberg multi-product duopoly game in which firms incur a higher fixed cost when offering different product lines in different quality tiers (mixed mode) than when offering all product lines in the same quality tier (high or low). To the best of our knowledge, this type of interdependence has not been analyzed in the previous literature on multi-product oligopoly, despite its real-world relevance. In our model, each firm can offer any subset of a given range of products that have identical and independent demands. Each product can be offered in one of two qualities (or modes) - high or low - and a firm that offers positive numbers of products in each mode incurs a fixed complexity cost $F \geq 0$. Firms compete product-by-product, so the only inter-dependence across products is driven by costs, not by demand.

We first show that both firms' profits are higher when the complexity cost is infinite (i.e. each firm must offer all of its products in the same mode) than when the complexity fixed cost is 0 (i.e. firms can costlessly use a mixed mode). Indeed, the inability to offer different products in different modes makes it easier for firms to sustain differentiation with respect to the offered products, so that each firm ends up being a monopolist on the products it offers. In contrast, when firms can use a mixed mode costlessly, the choices of mode (high or low) for individual products are independent of one another, so firms cannot avoid competing on each product as a vertical differentiated duopoly.

The determination of the equilibrium is more complex when the fixed cost is positive but finite. The Stackelberg leader's (firm 1's) profits are higher for all $F>0$ than for $F=0$, but they can be non-monotonic in $F$. The reason is that the fixed complexity cost can have two opposing effects on firm 1's profits: i) a direct, negative effect when firm 1 chooses a mixed mode strategy (i.e. offers some products in each mode), and ii) an indirect, positive effect, by lowering the profits that the Stackelberg follower (firm 2) can achieve with a mixed mode, thereby relaxing the constraints on firm 1 . On the other hand, the Stackelberg follower's profits can be lower when the complexity cost is positive and

\footnotetext{
${ }^{1}$ See W. Smit and C. Dula, "Singapore Airlines' Branding of its Low-Cost Carriers," Financial Times, March 26, 2014.

${ }^{2}$ See T. Hsu and W. Hamilton, "J.C. Penney Ourst CEO Ron Johnson, Brings Back His Predecessor," Los Angeles Times. April 9, 2013.

${ }^{3}$ See M. Maynard, "Delta to Close Song, its Low-Fare Airline," New York Times, October 29, 2005.
} 
finite than when it is equal to 0. Furthermore, consumer surplus is higher when the firms can freely offer products in multiple modes, since consumer surplus is higher in a differentiated duopoly than in a single quality monopoly market.

In most of the literature on multi-product monopoly and duopoly (e.g. Mussa and Rosen 1978, GalOr 1983, Champsaur and Rochet 1989, Klemperer 1992, and Johnson and Myatt 2003), the fixed costs of offering an additional quality version or product line are typically 0 (marginal costs are, however, allowed to depend on product quality). In contrast, the key feature of our model is the interdependence of firms' quality choices across product lines due to fixed costs. This type of interdependency has not previously been studied, despite its real-world relevance.

Furthermore, unlike most of the literature on multi-product monopoly and duopoly, which assumes all product lines are within the same product category and therefore substitutes, we focus on independent product lines. In other words, in our model consumer demand for each product line is independent of demand for the other product lines. Thus, we allow for two dimensions of differentiation between firms: vertical differentiation within the same product line and product line differentiation (i.e. firms may offer different product lines). A prominent exception to the single product category assumption is Klemperer (1992), which analyzes a circular Hotelling-type setting where duopolists are restricted to offering $N$ products at 0 fixed costs, but consumers have disutility from making purchases at multiple firms (e.g. switching costs). The difference is that in our model demands are independent across product lines, therefore our focus is on supply-side interdependencies, not on demand drivers as in Klemperer (1992).

The rest of the paper is organized as follows. In section 2, we present the basic model: we use a reduced form set-up that encompasses a large set of demand functions. We treat the baseline cases with complexity cost equal to 0 and infinity in section 3 . This allows us to establish some basic intuition and define two important strategies for the Stackelberg leader (full-spectrum and accommodation). Section 4 contains the analysis for all finite complexity cost. We analyze extensions in section 5 and conclude in the final section 6 .

\section{Model set-up}

There are two competing firms, 1 and 2. Each firm $i \in\{1,2\}$ can choose a continuous number of products $N_{i} \in[0, N]$ to offer and one of two possible modes, $\mathrm{L}$ or $\mathrm{H}$, for each product (in section 5.2 we allow firms to offer a product in both modes). Demands are identical and independent across products, i.e. competition occurs product by product. The two modes should be interpreted as corresponding to two vertically differentiated versions: high and low intrinsic qualities of the product or high and low levels of service accompanying the product.

If one of the two firms is a monopolist on any given product, its revenues net of marginal costs are $R_{H}$ if it offers the product in $\mathrm{H}$ mode and $R_{L}$ if it offers the product in $\mathrm{L}$ mode. If both firms offer 
a given product in the same mode, Bertrand competition with no differentiation implies that their respective revenues net of marginal costs are 0 . If firm $i$ offers a product in $\mathrm{H}$ mode, whereas firm $j$ offers it in $\mathrm{L}$ mode, then firm $i$ 's equilibrium revenues net of marginal costs are $r_{H}$, whereas firm $j$ 's are $r_{L}$. The fixed costs required to make one product available in $\mathrm{H}$ mode and, respectively, $\mathrm{L}$ mode are $f_{H}$ and $f_{L}$, where $f_{H} \geq f_{L} \geq 0$. Denote then the net profits corresponding to the revenues defined above as follows:

$$
\begin{aligned}
\Pi_{H} & \equiv R_{H}-f_{H} \\
\Pi_{L} & \equiv R_{L}-f_{L} \\
\pi_{H} & \equiv r_{H}-f_{H} \\
\pi_{L} & \equiv r_{L}-f_{L}
\end{aligned}
$$

We make the following two assumptions throughout.

Assumption $1 \quad \Pi_{H}>\Pi_{L}>\pi_{L}>0$ and $\Pi_{H}>\pi_{H}>\pi_{L}>0$.

Assumption 2 Demand for each product is such that a monopolist never finds it profitable to offer the product in both modes.

The first assumption simply ensures consistency with the interpretation of two vertically differentiated product versions: the $\mathrm{H}$ mode is more profitable than the $\mathrm{L}$ mode, even accounting for fixed costs. The second assumption rules out price discrimination reasons for offering multiple (vertically differentiated) versions of the same product - such price discrimination motivations are studied indepth by Johnson and Myatt (2003). This assumption implies that throughout most of the analysis neither firm has any incentive to offer any given product in both modes. The only exception is when a firm wishes to foreclose the other firm, a possibility we discuss in Section 5.2.

The following example satisfies both assumptions above. Consumers are indexed by $v$, uniformly distributed on $[0,1]$. Consumer $v$ 's willingness-to-pay for a product of quality $q$ is $q v$. The L mode corresponds to quality $q_{L}$ and the $\mathrm{H}$ mode corresponds to quality $q_{H}>q_{L}$. Assume 0 marginal costs. With this demand structure, profits are:

$$
\begin{aligned}
\Pi_{H} & =\frac{q_{H}}{4}-f_{H} \text { and } \Pi_{L}=\frac{q_{L}}{4}-f_{L} \\
\pi_{H} & =\frac{4 q_{H}^{2}\left(q_{H}-q_{L}\right)}{\left(4 q_{H}-q_{L}\right)^{2}}-f_{H} \text { and } \pi_{L}=\frac{q_{H} q_{L}\left(q_{H}-q_{L}\right)}{\left(4 q_{H}-q_{L}\right)^{2}}-f_{L}
\end{aligned}
$$

It is easily verified that this example satisfies both assumptions for any $\left(q_{H}, q_{L}\right)$, provided $f_{H}-f_{L}$ is not too large. ${ }^{4}$

It is worth emphasizing that $f_{L}$ and $f_{H}$ do not play any significant role in our analysis, which is why they are subsumed in the terms $\Pi_{H}, \Pi_{L}, \pi_{H}$ and $\pi_{L}$.

\footnotetext{
${ }^{4}$ Also, fixing $q_{L}$, there exists $q_{H}^{*}>q_{L}$ such that $\pi_{H}<\Pi_{L}$ for $q_{L}<q_{H}<q_{H}^{*}$ and $\pi_{H}>\Pi_{L}$ for $q_{H}>q_{H}^{*}$.
} 
On the other hand, the key ingredient of our model will be the fixed cost associated with offering products in both the $\mathrm{H}$ and $\mathrm{L}$ modes. To keep things as simple as possible, we assume that a firm that offers positive numbers of products in both modes incurs a "complexity fixed cost" $F \geq 0$. In other

words, this cost is incurred whenever a firm does not offer all of its products in the same mode. ${ }^{5}$ This fixed cost may correspond to the risk of confusing consumers by offering two vertically differentiated modes under the same brand or to the operational complexity of running two different business models. The fundamental effect of the complexity fixed cost is that it creates an interdependence between a firm's choices of modes across products.

The timing of the duopoly game in all scenarios is as follows:

1. Firm 1 chooses the respective numbers of products to offer in each mode, $N_{1}^{H}$ and $N_{1}^{L}$ such that $N_{1}^{H}+N_{1}^{L} \leq N$.

2. Firm 2 chooses the respective numbers of products to offer in each mode, $N_{2}^{H}$ and $N_{2}^{L}$ such that $N_{2}^{H}+N_{2}^{L} \leq N$.

3. Firms simultaneously compete in prices product by product and profits are realized.

Assuming the product selection stage precedes the pricing game is standard in the multi-product duopoly literature (see for example Champsaur and Rochet, 1989) and reflects the fact that product selection is typically a long-run decision, whereas prices can be modified more easily and frequently. It is then natural to also assume that firms choose their prices simultaneously.

There are two reasons for assuming Stackelberg timing in product selection. First, the Stackelberg timing reflects realistic scenarios in which firm 1 is an incumbent or industry leader, who makes product line choices first, whereas firm 2 is an entrant or a follower. Second, from a methodological standpoint, Stackelberg timing provides a natural way of ensuring a unique pure strategy equilibrium. In contrast, with Nash (simultaneous) timing, there will typically be multiple equilibria and sometimes there may only exist mixed strategy equilibria.

\section{Baseline results}

In this section, we derive the baseline results for two simple (and polar opposite) cases.

Suppose first that there is no complexity fixed cost associated with operating under both modes, i.e. $F=0$. This means that each firm's choices of modes are independent across products. In this case, the equilibrium is easily obtained.

Proposition 1 When firms' choices of modes are independent across products, there is a unique equilibrium in which firm 1 offers all $N$ products in $H$ mode and firm 2 offers all $N$ products in $L$ mode. Equilibrium profits are $N \pi_{H}$ for firm 1 and $N \pi_{L}$ for firm 2.

\footnotetext{
${ }^{5}$ In the extensions section we also analyze an alternative formulation, in which offering any positive number of products in $\mathrm{H}$ mode requires a "capability" fixed cost $F_{H} \geq 0$ and offering any positive number of products in $\mathrm{L}$ mode requires a "capability" fixed cost $F_{L} \geq 0$. The analysis is quite similar.
} 
Not surprisingly, since choices of modes across products are independent, this is the same (unique) equilibrium that would prevail if there was only one product. Firms offer vertically differentiated versions of each product, with firm 1 choosing the more profitable $\mathrm{H}$ mode and firm 2 choosing the $\mathrm{L}$ mode.

At the opposite extreme, suppose now that each firm must choose the same mode for all products it offers. This corresponds to the case when the complexity fixed cost is prohibitively high, i.e. $F \rightarrow \infty$. In this case, each firm chooses one of the two modes - $\mathrm{H}$ or $\mathrm{L}$ - and the number of products to offer in that mode. Thus, suppose firm 1 has chosen the $\mathrm{H}$ mode in stage 1 and $N_{1} \leq N$ products. Firm 2 has two options:

- Choose the L mode: clearly Firm 2 will offer all products in L mode, since $\pi_{L}>0$. Firm 2's equilibrium profits are then

$$
N_{1} \pi_{L}+\left(N-N_{1}\right) \Pi_{L}
$$

- Choose the H mode: firm 2 will only offer the $N-N_{1}$ products not offered by firm 1 . Firm 2's equilibrium profits are then

$$
\left(N-N_{1}\right) \Pi_{H}
$$

Comparing, firm 2 chooses the L mode if and only if

$$
N_{1} \pi_{L}+\left(N-N_{1}\right) \Pi_{L}>\left(N-N_{1}\right) \Pi_{H}
$$

which can also be written as:

$$
N_{1}>N \frac{\Pi_{H}-\Pi_{L}}{\Pi_{H}-\Pi_{L}+\pi_{L}} \equiv N_{H}^{*}
$$

Thus, if the number of products offered by firm 1 in $\mathrm{H}$ mode is larger than the threshold $N_{H}^{*}$, firm 2 responds by competing on all products with a vertically differentiated version. If, on the other hand, $N_{1}$ is below the threshold $N_{H}^{*}$ then firm 2 finds it profitable to not compete on the products offered by firm 1 , so that both firms end up with $\mathrm{H}$ mode monopoly profits on their respective products. The threshold is increasing in $\Pi_{H}$ and decreasing in $\Pi_{L}$ and $\pi_{L}$ : the higher the monopoly $\mathrm{H}$ profits and the lower L profits (monopoly and duopoly), the easier it is to induce firm 2 to be content with being an $\mathrm{H}$ monopolist on $N-N_{1}$ products instead of offering all products in L mode (which involves competing on the $N_{1}$ products offered by firm 1).

Stepping back to stage 1 , firm 1 profits are $N_{1} \Pi_{H}$ if $N_{1} \leq N_{H}^{*}$ and $N_{1} \pi_{H}$ if $N_{1}>N_{H}^{*}$. We conclude that when firm 1 chooses the $\mathrm{H}$ mode, its optimal profits are $\max \left\{N \pi_{H}, N_{H}^{*} \Pi_{H}\right\}$. In other words, firm 1 has a choice between duopoly profits on all relevant products and monopoly profits on a subset of these products. Relegating the rest of the proof to the appendix, we obtain the following proposition.

Proposition 2 When each firm must offer all products in the same mode, the duopoly equilibrium is as follows:

- If $\Pi_{H}-\Pi_{L} \geq \frac{\pi_{L} \pi_{H}}{\Pi_{H}-\pi_{H}}$ then firm 1 chooses the $H$ mode, offers $N_{1}=\frac{N\left(\Pi_{H}-\Pi_{L}\right)}{\Pi_{H}-\Pi_{L}+\pi_{L}}$ products and 
makes profits $\frac{N\left(\Pi_{H}-\Pi_{L}\right) \Pi_{H}}{\Pi_{H}-\Pi_{L}+\pi_{L}}$. Firm 2 offers the other $\frac{N \pi_{L}}{\Pi_{H}-\Pi_{L}+\pi_{L}}$ products in $H$ mode and makes profits $\frac{N \pi_{L} \Pi_{H}}{\Pi_{H}-\Pi_{L}+\pi_{L}}$.

- If $\Pi_{H}-\Pi_{L}<\frac{\pi_{L} \pi_{H}}{\Pi_{H}-\pi_{H}}$ then firm 1 chooses the $H$ mode, offers $N_{1}=N$ products and makes profits $N \pi_{H}$. Firm 2 offers all products in $L$ mode and makes profits $N \pi_{L}$.

Comparing Propositions 1 and 2 reveals that firm 1 makes (weakly) higher profits when firms are constrained to offer all products under the same mode. In particular, profits are strictly higher when $\Pi_{H}$ is sufficiently large relative to both $\Pi_{L}$ and $\pi_{H}$. Furthermore, firm 2 also makes (weakly) higher profits when $F=+\infty$. Indeed,

$$
\frac{N \pi_{L} \Pi_{H}}{\Pi_{H}-\Pi_{L}+\pi_{L}}>N \pi_{L}
$$

because $\Pi_{L}>\pi_{L}$.

The intuition behind this result is that the inability to choose different modes for different products allows the firms to also differentiate with respect to the products they offer. This can be a more effective form of differentiation than the vertical differentiation within each product, provided that monopoly $\mathrm{H}$ profits are sufficiently large relative to monopoly and duopoly L profits. Indeed, if monopoly L profits are too close to monopoly $\mathrm{H}$ profits, then firm 1 cannot induce firm 2 to offer a restricted number of $\mathrm{H}$ products instead of all products in $\mathrm{L}$ mode. Anticipating this, firm 1 has no choice but to resign itself to duopoly $\mathrm{H}$ profits on all products.

Finally, note that consumer welfare is strictly lower in the equilibrium in which firm 1 offers $N_{1}<N$ products in $\mathrm{H}$ mode and induces firm 2 to offer the remaining products in $\mathrm{H}$ mode as well. In this equilibrium, all products are offered, but each product is only offered by a monopolist. By contrast, in the equilibrium in which firm 1 offers all $N$ products in $\mathrm{H}$ mode, each products ends up being offered by a vertically differentiated duopoly, so consumer welfare is higher.

In what follows, we will refer to firm 1's strategy of offering all products in $\mathrm{H}$ mode as the "fullspectrum strategy" and to its strategy of only offering a limited number of products in $\mathrm{H}$ mode (thereby inducing firm 2 to only offer the other products in $\mathrm{H}$ mode) as the "accommodation strategy."

\section{Equilibrium with finite complexity fixed cost}

In this section, we study the case in which firms are not forced to use the same mode for all the products they offer, but they need to incur a finite complexity fixed cost $F>0$ if they offer positive numbers of products in each mode.

Denote:

$$
N_{H}^{*}(F) \equiv \min \left\{\frac{N\left(\Pi_{H}-\Pi_{L}\right)}{\Pi_{H}-\Pi_{L}+\pi_{L}}, \frac{F}{\pi_{L}},\right\}
$$

The number $N_{H}^{*}(F) \in[0, N]$ represents the maximum number of products that firm 1 can offer in $\mathrm{H}$ mode such that firm 2's best response is to only offer the remaining $N-N_{H}^{*}(F)$ products in $\mathrm{H}$ mode. The first term in the $\min \{\}$ operator (equal to $N_{H}^{*}(+\infty)$ ) is the same as the threshold in Proposition 2. This term is the maximum number of products that firm 1 can offer in $\mathrm{H}$ mode such 
that firm 2 prefers offering the remaining products in $\mathrm{H}$ mode as a monopolist to offering all products in $\mathrm{L}$ mode. The second term is new and represents the maximum number $N_{H}$ of products that firm 1 can offer in $\mathrm{H}$ mode such that firm 2 is content to be a monopolist in mode $\mathrm{H}$ on the remaining $N-N_{H}$ products and does not want to also compete in L mode on the $N_{H}$ products offered by firm 1 (this would yield additional profits $\pi_{L} N_{H}$ at cost $F$ ).

With this notation, the maximum profits that firm 1 can achieve with the accommodation strategy are $N_{H}^{*}(F) \Pi_{H}$. As before, these profits need to be compared with $N \pi_{H}$, the profits resulting from the full-spectrum strategy, but now firm 1 can also consider a mixed mode strategy, i.e. offering some products in $\mathrm{H}$ mode and others in $\mathrm{L}$ mode. The following proposition (proven in the appendix), provides the full characterization of firm 1's optimal strategy.

Proposition 3 When firms incur a fixed complexity cost $F>0$ for using a mixed mode, firm 1 may offer products in $H$ mode only or in both modes, firm 2 only offers products in a single mode and

- if $\frac{F}{N}<\frac{\pi_{L} \pi_{H}}{\pi_{L}+\pi_{H}}$ then firm 1's optimal profits are

$$
\max \left\{N \pi_{H}, N_{H}^{*}(F) \Pi_{H}, N \pi_{L}+\left(\frac{\Pi_{H}}{\pi_{L}}-2\right) F, N \pi_{H}+\left(\frac{\Pi_{L}}{\pi_{H}}-2\right) F\right\}
$$

- if $\frac{F}{N}>\frac{\pi_{L} \pi_{H}}{\pi_{L}+\pi_{H}}$ then firm 1's optimal profits are

$$
\max \left\{N \pi_{H}, N_{H}^{*}(F) \Pi_{H}, \frac{\left(\Pi_{H} \pi_{H}+\pi_{L}^{2}\right) N}{\pi_{L}+\pi_{H}}-F, \frac{\left(\Pi_{L} \pi_{L}+\pi_{H}^{2}\right) N}{\pi_{L}+\pi_{H}}-F\right\}
$$

Thus, when $F$ is sufficiently small, firm 1's optimal profits (from (1)) are simply $N \pi_{H}$, i.e. the same as in Proposition $1(F=0)$. In this case, firm 2's profits are $N \pi_{L}$. Meanwhile, when $F$ becomes large enough, the expression of firm 1's optimal profits (from (2)) becomes

$$
\max \left\{N \pi_{H}, \frac{N\left(\Pi_{H}-\Pi_{L}\right)}{\Pi_{H}-\Pi_{L}+\pi_{L}} \Pi_{H}\right\},
$$

i.e. the same as in Proposition $2(F=+\infty)$. The first two terms for both regions of fixed costs in Proposition 3 (expressions (1) and (2)) are simply the full-spectrum and accommodation strategy profits. The third and fourth terms for both regions correspond to mixed mode strategies for firm 1.

To understand the difference between the two cases in the proposition, it is first useful to note that in any equilibrium firm 1 precludes firm 2 from using a mixed mode strategy. Indeed, if firm 2 used a mixed mode strategy in equilibrium, then it would necessarily cover the entire product range, since it is already incurring the fixed cost $F$ and adding an additional product yields at least $\pi_{L}>0$. Denoting by $N_{2}$ the number of products offered in $\mathrm{H}$ mode by firm 2, we have $0<N_{2}<N$. In this equilibrium, firm 1's profits would be $N_{2} \pi_{L}$ or $\left(N-N_{2}\right) \pi_{H}$ or $N_{2} \pi_{L}+\left(N-N_{2}\right) \pi_{H}-F$. But each of these profits are dominated by $N \pi_{H}$, the profit that firm 1 can guarantee by choosing the full-spectrum strategy (all products in $\mathrm{H}$ mode). 
As discussed before the proposition, the necessity of preventing firm 2 from using a mixed mode strategy explains the new expression of $N_{H}^{*}(F)$ in firm 1's accommodation strategy. When firm 1 uses the accommodation strategy, firm 2's resulting profits are $\left(N-N_{H}^{*}(F)\right) \Pi_{H}$.

Focusing now on equilibria in which firm 1 chooses a mixed mode strategy, the difference between the two cases is whether or not the constraint of having to prevent firm 2 from using a mixed mode is binding. In the first case $\left(\frac{F}{N}<\frac{\pi_{L} \pi_{H}}{\pi_{L}+\pi_{H}}\right)$, the complexity fixed cost is sufficiently small, so firm 1 needs to take into account that firm 2 can profitably offer products in both modes. To prevent firm 2 from doing so, the number $N_{1}$ of products that firm 1 offers in $\mathrm{H}$ mode must either be small enough so that firm 2 does not want to offer those same products in L mode $\left(N_{1} \pi_{L}<F\right)$, or large enough so that firm 2 does not want to offer in $\mathrm{H}$ mode the same products that firm 1 offers in L mode $\left(\pi_{H}\left(N-N_{1}\right) \leq F\right)$. These two possibilities lead to the third and fourth terms in (1). The corresponding firm 2 profits are $\left(N-\frac{F}{\pi_{L}}\right) \pi_{H}$ when firm 1 chooses the third option in (1) and $\left(N-\frac{F}{\pi_{H}}\right) \pi_{L}$ when firm 1 chooses the fourth option in (1). In particular, note that when firm 1 chooses the fourth option, firm 2's profits are strictly lower than $N \pi_{L}$, the profits it makes when $F=0$.

In the second case, $\left(\frac{F}{N}>\frac{\pi_{L} \pi_{H}}{\pi_{L}+\pi_{H}}\right)$, the fixed cost incurred when adopting a mixed mode is sufficiently high that firm 2 never chooses the mixed mode option in response to firm 1 choosing the mixed mode strategy. Firm 1 still has two options with a mixed mode strategy:

- Induce firm 2 to only offer $\mathrm{H}$ products, specifically the $N-N_{1}$ products that firm 1 offers in L mode. This leaves firm 1 as an H-mode monopolist on $N_{1}$ products and an L-mode duopolist on $N-N_{1}$ products (third term in (2)). Firm 2's profits in this case are $\frac{N \pi_{H} \pi_{L}}{\pi_{L}+\pi_{H}}$, strictly lower than $N \pi_{L}$, the profits it makes when $F=0$.

- Induce firm 2 to only offer L products, specifically the $N_{1}$ products that 1 offers in $\mathrm{H}$ mode. This leaves firm 1 as an H-mode duopolist on $N_{1}$ products and an L-mode monopolist on $N-N_{1}$ products (fourth term in (2)). Firm 2's profits in this case are once again $\frac{N \pi_{L} \pi_{H}}{\pi_{L}+\pi_{H}}$.

It is interesting to discuss the effect of the fixed cost $F$ on firm 1's equilibrium profits.

Corollary 1 If $\frac{F}{N}<\frac{\pi_{L} \pi_{H}}{\pi_{L}+\pi_{H}}$ then firm 1's equilibrium profits are weakly increasing in $F$; if $\frac{F}{N}>\frac{\pi_{L} \pi_{H}}{\pi_{L}+\pi_{H}}$ then firm 1's equilibrium profits can be either increasing or decreasing in F.

Proof. Consider the case $\frac{F}{N}<\frac{\pi_{L} \pi_{H}}{\pi_{L}+\pi_{H}}$. The term $N_{H}^{*}(F) \Pi_{H}$ is weakly increasing in $F$ and the only way in which one of the mixed mode strategy profits in (1) dominates the baseline profit $N \pi_{H}$ is if $\frac{\Pi_{H}}{\pi_{L}}-2>0$ or $\frac{\Pi_{H}}{\pi_{L}}-2>0$, which means those profits are also increasing in $F$. If $\frac{F}{N}>\frac{\pi_{L} \pi_{H}}{\pi_{L}+\pi_{H}}$, then if firm 1 uses the accommodation strategy profits are increasing in $F$, while it if uses either mixed mode strategy it is falling in $F$.

When $F$ is not too large, it affects firm 1's profits both directly and indirectly. The direct effect is always negative: it reduces the profits that firm 1 can obtain with a mixed-mode strategy. In contrast, the indirect effect is positive: a larger $F$ reduces firm 2's profits from using a mixed mode, which in turn relaxes the constraints faced by firm 1 and therefore enhances (weakly) its profits. The negative 
direct effect only comes into play when firm 1 uses a mixed-mode strategy, but for such a strategy to be dominant, it is necessary that the positive indirect effect of $F$ on firm 1's profits outweighs the negative direct effect. Thus, firm 1's profits are weakly increasing in $F$ in this parameter range.

Consider now the case when $F$ is above the threshold $\frac{N \pi_{L} \pi_{H}}{\pi_{L}+\pi_{H}}$. If firm 1 chooses the accommodation strategy then its profits are weakly increasing in $F$ because $F$ only affects firm 1's profits indirectly: a larger $F$ relaxes the constraint of having to prevent firm 2 from using a mixed mode. On the other hand, if firm 1 chooses either one of the two mixed-mode strategies then $F$ only has the direct negative effect on its equilibrium profits since firm 2 would never consider choosing a mixed mode in this case. This is why the overall effect of $F$ on firm 1's profits is ambiguous on this parameter range.

Figures 1 and 2 illustrate the two possible cases emerging from this discussion. Both use the linear vertical differentiation example presented in Section 2 with $f_{L}=f_{H}=0$, but with different values of $\left(q_{L}, q_{H}\right)$. In figure $1\left(q_{L}=5\right.$ and $\left.q_{H}=9\right)$, firm 1 profits are everywhere weakly increasing in $F$. In figure $2\left(q_{L}=5\right.$ and $\left.q_{H}=5.25\right)$, firm 1 profits are non-monotonic in $F$.

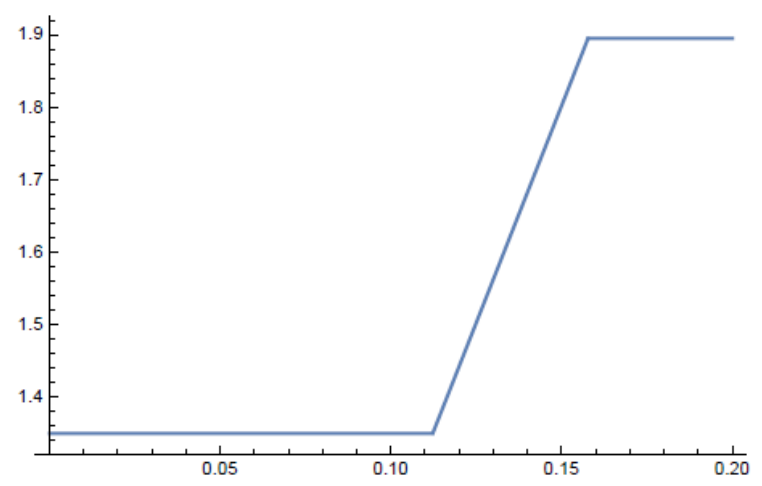

Figure 1

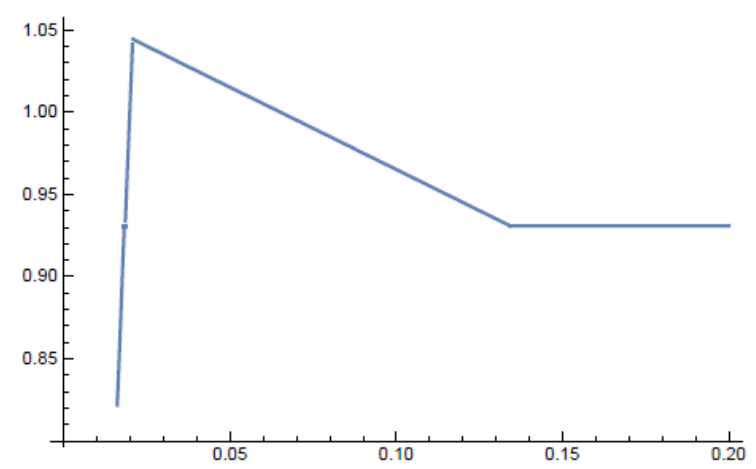

Figure 2 


\section{Extensions}

\subsection{Equilibrium with capability fixed costs}

An alternative way to introduce interdependence between a firm's choices of modes across products lines through fixed costs is to assume that each mode requires a fixed "capability" investment, instead of the complexity fixed cost studied above. Specifically, suppose that offering any positive number of products in $\mathrm{H}$ mode requires a fixed cost $F_{H} \geq 0$ and offering any positive number of products in $\mathrm{L}$ mode requires a fixed cost $F_{L} \geq 0$. We still maintain the assumption that the firm can only offer one mode per product. Consistent with the interpretation of vertical differentiation ( $\mathrm{H}$ corresponds to the higher quality mode), we assume the fixed cost required for the $\mathrm{H}$ capability is larger than the fixed cost required for the $\mathrm{L}$ capability:

$$
F_{H}>F_{L} .
$$

Furthermore, we also assume that fixed costs are not too high, such that both modes are viable for a monopolist as a well as for a duopolist:

$$
N \pi_{L}>F_{L} \text { and } N \pi_{H}>F_{H}
$$

Denote:

$$
\begin{aligned}
N_{H}^{*} & \equiv \min \left\{\frac{N\left(\Pi_{H}-\Pi_{L}\right)-\left(F_{H}-F_{L}\right)}{\Pi_{H}-\Pi_{L}+\pi_{L}}, \frac{F_{L}}{\pi_{L}}\right\} \\
N_{L}^{*} & \equiv\left\{\begin{array}{cc}
0 & \text { if } F_{H}-F_{L}-N\left(\Pi_{H}-\Pi_{L}\right) \leq 0 \\
\frac{F_{H}-F_{L}-N\left(\Pi_{H}-\Pi_{L}\right)}{\pi_{H}-\left(\Pi_{H}-\Pi_{L}\right)} & \text { if } F_{H}-F_{L}-N\left(\Pi_{H}-\Pi_{L}\right)>0
\end{array}\right.
\end{aligned}
$$

The expression of $N_{H}^{*}$ is very similar to $N_{H}^{*}(F)$ from section 4 and has the same meaning: it is the maximum number of products that firm 1 can offer in $\mathrm{H}$ mode such that firm 2's best response is to only offer the remaining $N-N_{H}^{*}$ products in H mode (accommodation strategy). Similarly, $N_{L}^{*}$ represents the maximum number of products that firm 1 can offer in $\mathrm{L}$ mode such that firm 2's best response is to only offer the remaining $N-N_{L}^{*}$ products in L mode. Note that $N_{L}^{*} \geq 0$ in all cases.

In the appendix, we derive the full equilibrium summarized in the following proposition.

Proposition 4 Suppose the firms incur fixed costs $F_{H}$ and $F_{L}$ for operating in $H$ mode and $L$ mode respectively.

- If $\frac{F_{L}}{\pi_{L}}+\frac{F_{H}}{\pi_{H}}<N$ then firm 1's optimal profits are

$$
\max \left\{\begin{array}{c}
N \pi_{H}-F_{H}, N \pi_{L}-F_{L}, \\
N_{H}^{*} \Pi_{H}-F_{H}, N_{L}^{*} \Pi_{L}-F_{L}, \\
N \pi_{L}+\left(\frac{\Pi_{H}}{\pi_{L}}-2\right) F_{L}-F_{H}, N \pi_{H}+\left(\frac{\Pi_{L}}{\pi_{H}}-2\right) F_{H}-F_{L}
\end{array}\right\}
$$


- If $\frac{F_{L}}{\pi_{L}}+\frac{F_{H}}{\pi_{H}}>N$ then firm 1's optimal profits are

$$
\max \left\{\begin{array}{c}
N \pi_{H}-F_{H}, N \pi_{L}-F_{L} \\
N_{H}^{*} \Pi_{H}-F_{H}, N_{L}^{*} \Pi_{L}-F_{L}, \\
N \Pi_{L}+\left(\Pi_{H}-\Pi_{L}\right) \frac{F_{L}}{\pi_{L}}-F_{H}-F_{L}
\end{array}\right\}
$$

The first two terms for both cases are the full-spectrum strategy profits. The difference with respect to Proposition 3 is that now firm 1 may find it profitable to offer the full spectrum of products under either the $\mathrm{H}$ mode or the $\mathrm{L}$ mode, depending on which option yields higher revenues net of fixed costs (in Proposition 3, the full-spectrum strategy in $\mathrm{H}$ mode always dominated the full spectrum strategy in L mode). The third and fourth terms in both (3) and (4) are the accommodation strategy profits. Once again, the difference with respect to Proposition 3 is that either the $\mathrm{H}$ or the $\mathrm{L}$ mode may be optimal for the accommodation strategy.

When $\frac{F_{L}}{\pi_{L}}+\frac{F_{H}}{\pi_{H}}<N$, the fifth and sixth terms in (3) correspond to mixed mode strategies for firm 1 and they are almost identical to the corresponding terms in Proposition 3 (save for the difference in the fixed cost structure). Their interpretation is the same: firm 1 needs to take into account that firm 2 can profitably enter with a mixed strategy. To prevent this, firm 1 must offer a number $N_{1}$ of products in $\mathrm{H}$ mode that is either small enough so that firm 2 does not want to offer those same products in L mode $\left(N_{1} \pi_{L}<F_{L}\right)$ or large enough so that firm 2 does not want to offer in $\mathrm{H}$ mode the products that firm 1 offers in L mode $\left(\left(N-N_{1}\right) \pi_{H}<F_{H}\right)$.

When $\frac{F_{L}}{\pi_{L}}+\frac{F_{H}}{\pi_{H}}>N$, firm 1 can foreclose firm 2 with a mixed mode strategy that denies firm 2 viable scale in both modes - a novel possibility relative to Proposition 3. Specifically, firm 1 can

offer $N_{1}=\frac{F_{L}}{\pi_{L}}$ products in $\mathrm{H}$ mode and $N-N_{1}<\frac{F_{H}}{\pi_{H}}$ in L mode: as a result, firm 2 cannot make positive profits in either mode $\left(\pi_{L} N_{1}=F_{L}\right.$ and $\left.\pi_{H}\left(N-N_{1}\right)<F_{H}\right)$. Furthermore, it turns out that the profits that firm 1 can obtain with this foreclosure strategy dominate the maximum profits it can obtain with any other mixed strategy that allows firm 2 to enter. This is why in this case the only options to consider for firm 1 are full-spectrum, accommodation and foreclosure (fifth term in (4)).

\section{$5.2 \quad$ Foreclosure}

Throughout the previous analysis, we have assumed that neither firm can offer a product in both modes. Assumption 2 guarantees that firm 2 never has an incentive to do so. On the other hand, firm 1 may find it profitable to offer a product in both modes, but only if it leads to foreclosure of firm 2. Consider the main model above with fixed complexity costs $F$. Allowing firm 1 to foreclose simply amounts to adding a fifth option to the ones described in the text of Proposition 3. Foreclosure yields profits for firm 1 equal to

$$
N\left(\Pi_{H}-f_{L}\right)-F
$$

Indeed, this dominates any strategy in which firm 1 would only offer a subset $n<N$ products in both modes. To see why, note that assumption 2 implies that $R_{H} \geq r_{H}+r_{L}$, which together with $r_{L}>f_{L}$ (assumption 1) leads to $\Pi_{H}-f_{L}>\pi_{H}$. This means that the highest profits firm 1 can hope to achieve 
by only offering $n<N$ products in both modes are

$$
n\left(\Pi_{H}-f_{L}\right)-F+(N-n) \pi_{H}<N\left(\Pi_{H}-f_{L}\right)-F .
$$

Thus, firm 1's optimal profits from Proposition 3 become

$$
\left\{\begin{array}{cll}
\max \left\{N\left(\Pi_{H}-f_{L}\right)-F, N \pi_{H}, N_{H}^{*}(F) \Pi_{H}, N \pi_{L}+\left(\frac{\Pi_{H}}{\pi_{L}}-2\right) F, N \pi_{H}+\left(\frac{\Pi_{L}}{\pi_{H}}-2\right) F\right\} & \text { if } \frac{F}{N}<\frac{\pi_{L} \pi_{H}}{\pi_{L}+\pi_{H}} \\
\max \left\{N\left(\Pi_{H}-f_{L}\right)-F, N \pi_{H}, N_{H}^{*}(F) \Pi_{H}, \frac{\left(\Pi_{H} \pi_{H}+\pi_{L}^{2}\right) N}{\pi_{L}+\pi_{H}}-F, \frac{\left(\Pi_{L} \pi_{L}+\pi_{H}^{2}\right) N}{\pi_{L}+\pi_{H}}-F\right\} & \text { if } \frac{F}{N}>\frac{\pi_{L} \pi_{H}}{\pi_{L}+\pi_{H}}
\end{array}\right.
$$

It is clear from these expressions that foreclosure is not necessarily optimal, so most of the analysis in section 4 remains valid. Note that foreclosure is more likely when both $f_{L}$ and $F$ are smaller.

\section{Conclusion}

Many multi-product firms incur additional fixed costs when attempting to offer different products in different quality or service tiers. These costs are usually associated with brand confusion or the necessity of maintaining different brands, as well as with the operational complexity (diseconomies of scope) of running different business models under the same organization. Such complexity fixed costs create interdependencies in firms' choices of quality or service tiers across different product lines. We have shown that these cost-driven interdependencies create interesting and novel effects on the equilibria that arise in a Stackelberg duopoly model. The Stackelberg leader always benefits from positive complexity fixed costs (relative to the case with no complexity costs), but its profits can be non-monotonic in the magnitude of the complexity costs. In contrast, the Stackelberg follower's profits can be lower when complexity fixed costs are positive and finite, although they are (weakly) higher when the complexity fixed cost is infinite (i.e. when each firm must choose a single quality tier for all of the products it offers).

There are several promising avenues for extending our analysis. First, one could investigate the impact of complexity fixed costs on firm profits in the scenario when consumers incur switching costs between firms, so they prefer buying all products from the same firm (in our analysis above, consumer demands are independent across products). Second, one could introduce heterogeneity in product demands and investigate whether the Stackelberg leader would always offer the most profitable products or sometimes prefer to accommodate the follower by leaving some profitable products out of its line-up in order to reduce the follower's incentives to compete with the leader.

\section{References}

[1] Casadesus-Masanell, R. and F. Zhu (2010) "Strategies to Fight Ad-Sponsored Rivals," Management Science, 56 (9), 1484-1499. 
[2] Champsaur, P. and J.-C. Rochet (1989) "Multiproduct Duopolists," Econometrica, 57 (3), 533-557.

[3] Gal-Or, E. (1983) "Quality and Quantity Competition," Bell Journal of Economics, 14 (2), 590600 .

[4] Johnson, J. P. and D. P. Myatt (2003) "Multiproduct Quality Competition: Fighting Brands and Product Line Pruning," American Economic Review, 93 (3), 748-774.

[5] Klemperer, P. (1992) "Competing Product Lines: Competing Head-to-Head May Be Less Competitive," American Economic Review, 82 (4), 740-755.

[6] Mussa, M. and S. Rosen (1978) "Monopoly and Product Quality," Journal of Economic Theory, $18(2), 301-317$.

[7] Rivkin, J. (2006) "Delta Air Lines (A): The Low-Cost Carrier Threat and Delta Air Lines (B): The Launch of Song," Harvard Business School Teaching Note \#5-706-430.

[8] Shaked A. and J. Sutton (1982) "Relaxing Price Competition Through Product Differentiation," Review of Economic Studies, 49 (1), 3-13.

\section{Appendix}

\section{Proof of Proposition 2}

Suppose firm 1 has chosen the $\mathrm{H}$ mode in stage 1 and offers $N_{1} \leq N$ products. Firm 2 has two options:

- choose the L mode, in which case it is optimal to offer all $N$ products, leading to profits

$$
N_{1} \pi_{L}+\left(N-N_{1}\right) \Pi_{L}
$$

- choose the $\mathrm{H}$ mode, in which case it is optimal to only offer the $N-N_{1}$ products not offered by firm 1 , leading to profits

$$
\left(N-N_{1}\right) \Pi_{H}
$$

Comparing, firm 2 chooses the L mode (first option) if and only if

$$
N_{1} \pi_{L}+\left(N-N_{1}\right) \Pi_{L} \geq\left(N-N_{1}\right) \Pi_{H}
$$

which can also be written:

$$
N_{1} \geq N \frac{\Pi_{H}-\Pi_{L}}{\Pi_{H}-\Pi_{L}+\pi_{L}} \equiv N_{H}^{*}
$$

Stepping back to stage 1 , firm 1 profits are $N_{1} \Pi_{H}$ if $N_{1} \leq N_{H}^{*}$ and $N_{1} \pi_{H}$ if $N_{1}>N_{H}^{*}$. We conclude that when firm 1 chooses the $\mathrm{H}$ mode, its optimal profits are $\max \left\{N \pi_{H}, N_{H}^{*} \Pi_{H}\right\}$.

Suppose now firm 1 chooses the L mode and offers $N_{1} \leq N$ products. Again, firm 2 has two options: 
- choose the $\mathrm{H}$ mode, in which case it is optimal to offer all $N$ products, leading to profits

$$
N_{1} \pi_{H}+\left(N-N_{1}\right) \Pi_{H}
$$

- choose the L mode, in which case it is optimal to only offer the $N-N_{1}$ products not offered by firm 1 , leading to profits

$$
\left(N-N_{1}\right) \Pi_{L}
$$

Clearly, firm 2 always prefers the first option (recall $\Pi_{H}>\Pi_{L}$ ) so firm 1's profits are $N_{1} \pi_{L}$. But these profits are always smaller than what firm 1 can obtain by offering all products in the $\mathrm{H}$ mode (at least $N \pi_{H}$ ).

We can therefore conclude that firm 1 always chooses the $\mathrm{H}$ mode in equilibrium, earning profits $\max \left\{N \pi_{H}, N_{H}^{*} \Pi_{H}\right\}$. Comparing, $N \pi_{H} \geq N_{H}^{*} \Pi_{H}$ if and only if $\frac{\pi_{H} \pi_{L}}{\Pi_{H}-\pi_{H}} \geq \Pi_{H}-\Pi_{L}$.

\section{Proof of Proposition 3}

Firm 1 has three options: offer only H products, offer only L products or offer products in both modes. Let us examine each of these options in turn.

Option 1: firm 1 offers $N_{1}$ products in $\mathbf{H}$ mode, $0<N_{1} \leq N$

Firm 2 has three options in response:

- Offer H products only, in which case it is optimal to offer all $N-N_{1}$ products not offered by firm 1 in $\mathrm{H}$ mode. This yields profits $\left(N-N_{1}\right) \Pi_{H}$ for firm 2 and $N_{1} \Pi_{H}$ for firm 1.

- Offer L products only, in which case it is optimal to offer all products in L mode. This yields profits $\left(N-N_{1}\right) \Pi_{L}+N_{1} \pi_{L}$ for firm 2 and $N_{1} \pi_{H}$ for firm 1.

- Offer products in both modes, in which case it is optimal to offer all $N-N_{1}$ products not offered by firm 1 in $\mathrm{H}$ mode and the $N_{1}$ products offered by firm 1 in L mode. This yields profits $\left(N-N_{1}\right) \Pi_{H}+N_{1} \pi_{L}-F$ for firm 2 and $N_{1} \pi_{H}$ for firm 1 .

If firm 2 chooses one of the last two options, then this can be part of an equilibrium for firm 1 only if $N_{1}=N$, resulting in profits $N \pi_{H}$. Thus, the only way in which $N_{1}<N$ can be optimal for firm 1 is for firm 2 to prefer the first option over the other two. In this case, firm 1 chooses the largest $N_{1}$ such that $\left(N-N_{1}\right)\left(\Pi_{H}-\Pi_{L}\right) \geq N_{1} \pi_{L}$ and $N_{1} \pi_{L} \leq F$, i.e.

$$
N_{1}=N_{H}^{*}(F) \equiv \min \left\{\frac{F}{\pi_{L}}, \frac{N\left(\Pi_{H}-\Pi_{L}\right)}{\Pi_{H}-\Pi_{L}+\pi_{L}}\right\}
$$

Thus, the best profits that firm 1 can achieve with this option (products in $\mathrm{H}$ mode only) are

$$
\max \left\{N \pi_{H}, N_{H}^{*}(F) \Pi_{H}\right\}
$$

Option 2: firm 1 offers $N_{1}$ products in $\mathbf{L}$ mode, $0<N_{1} \leq N$

Firm 2 has the following three options in response: 
- Offer H products only, in which case it is optimal to offer all $N$ products in $\mathrm{H}$ mode. This yields profits $\left(N-N_{1}\right) \Pi_{H}+N_{1} \pi_{H}$ for firm 2 and $N_{1} \pi_{L}$ for firm 1.

- Offer L products only, in which case it is optimal to offer all $N-N_{1}$ products not offered by firm 1 in L mode. This yields profits $\left(N-N_{1}\right) \Pi_{L}$ for firm 2 .

- Offer products in both modes, in which case it is optimal to offer all $N-N_{1}$ products not offered by firm 1 in L mode and the $N_{1}$ products offered by firm 1 in $\mathrm{H}$ mode. This yields profits $\left(N-N_{1}\right) \Pi_{L}+N_{1} \pi_{H}-F$ for firm 2 .

It is immediately clear that firm 2 always prefers the first option, which means that the maximum profit that firm 1 can achieve in this scenario is $N \pi_{L}$. But this is dominated by the first option above: offering all products in $\mathrm{H}$ mode guarantees profits $N \pi_{H}>N \pi_{L}$. Therefore, this option will never be chosen by firm 1 in equilibrium.

Option 3: firm 1 offers $N_{1}$ products in $\mathbf{H}$ mode and $N-N_{1}$ products in $\mathbf{L}$ mode, $0<N_{1}<N$.

Indeed, it would be a dominated strategy for firm 1 to offer products in both modes without covering the entire available product range.

Firm 2 has the following three options in response:

- Offer H products only, in which case it is optimal to offer only the $N-N_{1}$ products that firm 1 offers in L mode. This yields profits $\left(N-N_{1}\right) \pi_{H}$ for firm 2 and $N_{1} \Pi_{H}+\left(N-N_{1}\right) \pi_{L}-F$ for firm 1.

- Offer L products only, in which case it is optimal to offer all $N_{1}$ products that firm 1 offers in $\mathrm{H}$ mode. This yields profits $N_{1} \pi_{L}$ for firm 2 and $N_{1} \pi_{H}+\left(N-N_{1}\right) \Pi_{L}-F$ for firm 1.

- Offer products in both modes, in which case it is optimal to offer each product in the other mode relative to firm 1. This yields profits $N_{1} \pi_{L}+\left(N-N_{1}\right) \pi_{H}-F$ for firm 2 and $N_{1} \pi_{H}+\left(N-N_{1}\right) \pi_{L}-F$ for firm 1.

Note that if firm 2 chooses the third option then firm 1's profits are dominated by what it can obtain with the first option above (all products in $\mathrm{H}$ mode, guaranteeing $N \pi_{H}$ ). Thus, it can never be optimal for firm 1 to induce firm 2 to choose its third option. In other words, firm 1 must choose $N_{1}$ here such that $N_{1} \pi_{L} \leq F$ or $\left(N-N_{1}\right) \pi_{H} \leq F$, i.e.

$$
N_{1} \leq \frac{F}{\pi_{L}} \text { or } N_{1} \geq N-\frac{F}{\pi_{H}}
$$

There are two cases to consider.

Suppose first $\frac{F}{\pi_{L}} \leq N-\frac{F}{\pi_{H}}$, i.e. $\frac{F}{N} \leq \frac{\pi_{L} \pi_{H}}{\pi_{L}+\pi_{H}}$. In this case, firm 1 must not choose $N_{1} \in\left(\frac{F}{\pi_{L}}, N-\frac{F}{\pi_{H}}\right)$ in order to avoid a mixed mode response by firm 2. If $N_{1} \leq \frac{F}{\pi_{L}}$ then firm 2 offers only $\mathrm{H}$ products, so firm 1 makes profits $N_{1} \Pi_{H}+\left(N-N_{1}\right) \pi_{L}-F$, which are clearly increasing in $N_{1}$. The best that firm 1 can achieve conditional on $N_{1} \leq \frac{F}{\pi_{L}}$ is then

$$
N \pi_{L}+\left(\frac{\Pi_{H}}{\pi_{L}}-2\right) F
$$


On the other hand, if $N_{1} \geq N-\frac{F}{\pi_{H}}$ then firm 2 offers only L products, so firm 1 makes profits $N_{1} \pi_{H}+$ $\left(N-N_{1}\right) \Pi_{L}-F$. If $\Pi_{L} \leq \pi_{H}$ then these profits are dominated by firm 1's option 1 (which guarantees at least $N \pi_{H}$ ). Thus, for this scenario to have a chance of yielding higher profits for firm 1 it must be that $\Pi_{L}>\pi_{H}$, in which case the best that firm 1 can achieve conditional on $N_{1} \geq N-\frac{F}{\pi_{H}}$ is

$$
N \pi_{H}+\left(\frac{\Pi_{L}}{\pi_{H}}-2\right) F
$$

Thus, firm 1's optimal profits when $\frac{F}{N} \leq \frac{\pi_{L} \pi_{H}}{\pi_{L}+\pi_{H}}$ are

$$
\max \left\{N \pi_{H}, N_{H}^{*}(F) \Pi_{H}, N \pi_{L}+\left(\frac{\Pi_{H}}{\pi_{L}}-2\right) F, N \pi_{H}+\left(\frac{\Pi_{L}}{\pi_{H}}-2\right) F\right\}
$$

Consider now the other case: $\frac{F}{N}>\frac{\pi_{L} \pi_{H}}{\pi_{L}+\pi_{H}}$, i.e. $N-\frac{F}{\pi_{H}}<\frac{F}{\pi_{L}}$. In this case it is never optimal for firm 2 to choose the mixed mode option in response to firm 1 choosing it. In particular, it is easily seen that firm 2 offers only $\mathrm{H}$ products when $N_{1} \leq \frac{N \pi_{H}}{\pi_{L}+\pi_{H}}$ and only L products only when $N_{1} \geq \frac{N \pi_{H}}{\pi_{L}+\pi_{H}}$. Consequently, firm 1 's profits are

$$
\begin{cases}N_{1} \Pi_{H}+\left(N-N_{1}\right) \pi_{L}-F & \text { for } N_{1}<\frac{N \pi_{H}}{\pi_{L}+\pi_{H}} \\ N_{1} \pi_{H}+\left(N-N_{1}\right) \Pi_{L}-F & \text { for } N_{1}>\frac{N \pi_{H}}{\pi_{L}+\pi_{H}}\end{cases}
$$

Since $N_{1} \Pi_{H}+\left(N-N_{1}\right) \pi_{L}-F$ is increasing in $N_{1}$, the best that firm 1 can achieve conditional on $N_{1}<\frac{N \pi_{H}}{\pi_{L}+\pi_{H}}$ is (slightly less than) $\frac{N\left(\Pi_{H} \pi_{H}+\pi_{L}^{2}\right)}{\pi_{L}+\pi_{H}}-F$. Conditional on $N_{1}>\frac{N \pi_{H}}{\pi_{L}+\pi_{H}}$, the best that firm 1 can achieve is either $\frac{N\left(\Pi_{L} \pi_{L}+\pi_{H}^{2}\right)}{\pi_{L}+\pi_{H}}-F$ or $N \pi_{H}-F$ (depending on whether $\pi_{H}<>\Pi_{L}$ ). But $N \pi_{H}-F$ is dominated by firm 1's option 1, which guarantees at least $N \pi_{H}$.

Consequently, firm 1's optimal profits when $\frac{F}{N}<\frac{\pi_{L} \pi_{H}}{\pi_{L}+\pi_{H}}$ are

$$
\max \left\{N \pi_{H}, N_{H}^{*}(F) \Pi_{H}, \frac{N\left(\Pi_{H} \pi_{H}+\pi_{L}^{2}\right)}{\pi_{L}+\pi_{H}}-F, \frac{N\left(\Pi_{L} \pi_{L}+\pi_{H}^{2}\right)}{\pi_{L}+\pi_{H}}-F\right\}
$$

\subsection{Proof of Proposition 4}

Suppose first firm 1 chooses the pure $\mathrm{H}$ mode, with $N_{1} \leq N$ products offered in $\mathrm{H}$ mode. Then firm 2's best three options in response are:

- pure $\mathrm{H}$ mode, yielding $\left(N-N_{1}\right) \Pi_{H}-F_{H}$ for firm 2 and $N_{1} \Pi_{H}-F_{H}$ for firm 1

- pure L mode, yielding $N_{1} \pi_{L}+\left(N-N_{1}\right) \Pi_{L}-F_{L}$ for firm 2 and $N_{1} \pi_{H}-F_{H}$ for firm 1

- hybrid HL mode, yielding $N_{1} \pi_{L}+\left(N-N_{1}\right) \Pi_{H}-F_{L}-F_{H}$ for firm 2 and $N_{1} \pi_{H}-F_{H}$ for firm 1

Note that the second option yields positive profits for firm 2 (recall $N \pi_{L}>F_{L}$ ), so firm 1 cannot foreclose firm 2 with a pure $\mathrm{H}$ strategy. For this to be an equilibrium with $N_{1}<N$, firm 2 must prefer the first option 
above, i.e. we must have

$$
N_{1} \leq \frac{F_{L}}{\pi_{L}} \text { and } N_{1} \leq \frac{N\left(\Pi_{H}-\Pi_{L}\right)-\left(F_{H}-F_{L}\right)}{\Pi_{H}-\Pi_{L}+\pi_{L}}
$$

which is equivalent to

$$
N_{1} \leq N_{H}^{*} \equiv \min \left\{\frac{N\left(\Pi_{H}-\Pi_{L}\right)-\left(F_{H}-F_{L}\right)}{\Pi_{H}-\Pi_{L}+\pi_{L}}, \frac{F_{L}}{\pi_{L}}\right\}
$$

If $N_{1}>N_{H}^{*}$ then firm 1 is better off offering all $N$ products in $\mathrm{H}$ mode.

Thus, the highest profits that firm 1 can obtain with a pure $\mathrm{H}$ strategy are $\max \left\{N \pi_{H}, N_{H}^{*} \Pi_{H}\right\}-F_{H}$.

Suppose next firm 1 chooses the pure L mode, with $N_{1} \leq N$ products offered in L mode. Then firm 2's best three options in response are:

- pure $\mathrm{H}$ mode, yielding $N_{1} \pi_{H}+\left(N-N_{1}\right) \Pi_{H}-F_{H}$ for firm 2 and $N_{1} \pi_{L}-F_{L}$ for firm 1

- pure L mode, yielding $\left(N-N_{1}\right) \Pi_{L}-F_{L}$ for firm 2 and $N_{1} \Pi_{L}-F_{L}$ for firm 1

- hybrid HL mode, yielding $N_{1} \pi_{H}+\left(N-N_{1}\right) \Pi_{L}-F_{L}-F_{H}$ for firm 2 and $N_{1} \pi_{L}-F_{L}$ for firm 1

Clearly, the third option is always dominated by the first option for firm 2, so firm 2 chooses the best among the first two options. For this to be an equilibrium with $N_{1}<N$, firm 2 must prefer the second option above, i.e. we must have

$$
N_{1}\left(\pi_{H}-\left(\Pi_{H}-\Pi_{L}\right)\right) \leq F_{H}-F_{L}-N\left(\Pi_{H}-\Pi_{L}\right)
$$

Given that $N \pi_{H}>F_{H}$, it can be verified that this inequality can only be satisfied if $F_{H}-F_{L}-$ $N\left(\Pi_{H}-\Pi_{L}\right)>0$, in which case it is equivalent to

$$
N_{1} \leq \frac{F_{H}-F_{L}-N\left(\Pi_{H}-\Pi_{L}\right)}{\pi_{H}-\left(\Pi_{H}-\Pi_{L}\right)}
$$

Thus, the highest profits that firm 1 can obtain with a pure $L$ strategy are $\max \left\{N \pi_{L}, N_{L}^{*} \Pi_{L}\right\}-F_{L}$, where

$$
N_{L}^{*} \equiv\left\{\begin{array}{cl}
0 & \text { if } F_{H}-F_{L}-N\left(\Pi_{H}-\Pi_{L}\right) \leq 0 \\
\frac{F_{H}-F_{L}-N\left(\Pi_{H}-\Pi_{L}\right)}{\pi_{H}-\left(\Pi_{H}-\Pi_{L}\right)} & \text { if } F_{H}-F_{L}-N\left(\Pi_{H}-\Pi_{L}\right)>0
\end{array}\right.
$$

Finally, suppose firm 1 chooses a hybrid mode, i.e. offers $N_{1}$ products in $\mathrm{H}$ mode and $N-N_{1}$ products in L mode, where $0<N_{1}<N$. Firm 2's best three response options are:

- pure H, yielding $\left(N-N_{1}\right) \pi_{H}-F_{H}$ for firm 2 and $N_{1} \Pi_{H}+\left(N-N_{1}\right) \pi_{L}-F_{H}-F_{L}$ for firm 1

- pure L, yielding $N_{1} \pi_{L}-F_{L}$ for firm 2 and $N_{1} \pi_{H}+\left(N-N_{1}\right) \Pi_{L}-F_{H}-F_{L}$ for firm 1

- hybrid HL, yielding $N_{1} \pi_{L}+\left(N-N_{1}\right) \pi_{H}-F_{L}-F_{H}$ for firm 2 and $N_{1} \pi_{H}+\left(N-N_{1}\right) \pi_{L}-F_{H}-F_{L}$ for firm 1 
The profits obtained by firm 1 under the third option are strictly lower than what firm 1 can obtain with the pure $\mathrm{H}$ strategy (at least $N \pi_{H}-F_{H}$ ), therefore it cannot be an equilibrium for firm 1 to choose the HL strategy and induce firm 2 to choose the third option above. Thus, we must have $\left(N-N_{1}\right) \pi_{H} \leq F_{H}$ or $N_{1} \pi_{L} \leq F_{L}$, i.e.

$$
N_{1} \leq \frac{F_{L}}{\pi_{L}} \text { or } N_{1} \geq N-\frac{F_{H}}{\pi_{H}}
$$

If both inequalities hold then firm 2 cannot make positive profits, so it is foreclosed. This is possible if and only if $N \leq \frac{F_{H}}{\pi_{H}}+\frac{F_{L}}{\pi_{L}}$.

Suppose $N \leq \frac{F_{H}}{\pi_{H}}+\frac{F_{L}}{\pi_{L}}$. Then firm can foreclose firm 2 by choosing $N_{1} \in\left[N-\frac{F_{H}}{\pi_{H}}, \frac{F_{L}}{\pi_{L}}\right]$, leading to profits $N_{1} \Pi_{H}+\left(N-N_{1}\right) \Pi_{L}-F_{H}-F_{L}$, which are increasing in $N_{1}$. As a result, the best foreclosure strategy for firm 1 is $N_{1}=\frac{F_{L}}{\pi_{L}}$, leading to firm 1 foreclosure profits

$$
\frac{F_{L}}{\pi_{L}} \Pi_{H}+\left(N-\frac{F_{L}}{\pi_{L}}\right) \Pi_{L}-F_{H}-F_{L}
$$

There are two other non-foreclosure possibilities for firm 1:

- $N_{1}>\frac{F_{L}}{\pi_{L}} \geq N-\frac{F_{H}}{\pi_{H}}$, which results in profits $N_{1} \pi_{H}+\left(N-N_{1}\right) \Pi_{L}-F_{H}-F_{L}$, maximized for either $N_{1}=N$ or $N_{1}=\frac{F_{L}}{\pi_{L}}$.

- $N_{1}<N-\frac{F_{H}}{\pi_{H}} \leq \frac{F_{L}}{\pi_{L}}$, which results in profits $N_{1} \Pi_{H}+\left(N-N_{1}\right) \pi_{L}-F_{H}-F_{L}$, maximized for $N_{1}=N-\frac{F_{H}}{\pi_{H}}$.

It is easily verified that the foreclosure profits dominate both of these possibilities, so foreclosure is the optimal strategy for firm 1 when it is feasible, i.e. when $N \leq \frac{F_{H}}{\pi_{H}}+\frac{F_{L}}{\pi_{L}}$.

Suppose now $N>\frac{F_{H}}{\pi_{H}}+\frac{F_{L}}{\pi_{L}}$. Then firm 1 has two options:

- $N_{1}<\frac{F_{L}}{\pi_{L}}<N-\frac{F_{H}}{\pi_{H}}$, which results in profits $N_{1} \Pi_{H}+\left(N-N_{1}\right) \pi_{L}-F_{H}-F_{L}$, maximized for $N_{1}=\frac{F_{L}}{\pi_{L}}$ (slightly below), which yields $N \pi_{L}+\left(\frac{\Pi_{H}}{\pi_{L}}-2\right) F_{L}-F_{H}$

- $N_{1}>N-\frac{F_{H}}{\pi_{H}}>\frac{F_{L}}{\pi_{L}}$, which results in profits $N_{1} \pi_{H}+\left(N-N_{1}\right) \Pi_{L}-F_{H}-F_{L}$. These profits are maximized either by $N_{1}=N$ or by $N_{1}=N-\frac{F_{H}}{\pi_{H}}$. In the first case, profits are clearly dominated by the pure $\mathrm{H}$ mode $\left(N \pi_{H}-F_{H}\right)$, so only the second case is relevant.

Thus, when $N>\frac{F_{H}}{\pi_{H}}+\frac{F_{L}}{\pi_{L}}$, the best profits that firm 1 can achieve with the HL mode are

$$
\max \left\{N \pi_{L}+\left(\frac{\Pi_{H}}{\pi_{L}}-2\right) F_{L}-F_{H}, N \pi_{H}+\left(\frac{\Pi_{L}}{\pi_{H}}-2\right) F_{H}-F_{L}\right\}
$$

